\title{
Chinese herbal medicine use in Taiwan during pregnancy and the postpartum period: A population-based cohort study
}

\author{
Chao-Hua Chuang ${ }^{a}$, Pei-Jen Chang ${ }^{b}$, Wu-Shiun Hsieh ${ }^{c}$, Yih-Jian Tsai ${ }^{d}$, \\ Shio-Jean Lin ${ }^{\mathrm{e}}$, Pau-Chung Chen ${ }^{\mathrm{f}, *}$ \\ ${ }^{a}$ Department of Nursing, Chang Jung Christian University, Tainan County, Taiwan \\ ${ }^{\mathrm{b}}$ Department of Nursing, National Taipei College of Nursing, Taipei, Taiwan \\ ${ }^{\mathrm{c}}$ Department of Pediatrics, National Taiwan University Hospital and National Taiwan University College of Medicine, Taipei, Taiwan \\ ${ }^{\mathrm{d}}$ Division of Health Promotion for Children and Adolescents, Bureau of Health Promotion, Department of Health, Taichung, Taiwan \\ e Department of Pediatrics, National Cheng Kung University Hospital and National Cheng Kung University College of Medicine, Tainan, Taiwan \\ ${ }_{\mathrm{f}}^{\mathrm{f}}$ Institute of Occupational Medicine and Industrial Hygiene, National Taiwan University College of Public Health, 17 Syujhou Road, Taipei 10055, Taiwan
}

\section{A R T I C L E I N F O}

\section{Article history:}

Received 8 July 2008

Received in revised form 31 October 2008

Accepted 28 December 2008

\section{Keywords:}

Chinese herbal medicine

Pregnancy

Postpartum

Prevalence rate

Risk factors

\begin{abstract}
A B S T R A C T
Background: Using Chinese herbal medicines during pregnancy and postpartum is common in the Chinese community.

Objective: The purpose of this current study is to explore the use of Chinese herbal medicines by women during pregnancy and postpartum in Taiwan.

Design: It is an on-going prospective longitudinal study design.

Setting and participants: We used multistage stratified systematic sampling to recruit 24,200 pairs, postpartum women and newborns, from the Taiwan national birth register in 2005. Subjects underwent a home interview 6 months after their deliveries between June 2005 and July 2006. A structured questionnaire was successfully administered to $87.8 \%$ of the sampled population.

Results: At least one Chinese herbal medicine was used by $33.6 \%$ and $87.7 \%$ of the interviewed subjects during pregnancy and the postpartum period, respectively. An-TaiYin, Pearl powder, and Huanglian were the most commonly used during pregnancy, while Shen-Hua-Tang and Suz-Wu-Tang were the most commonly used by postpartum women. Pregnant women aged 20-34, with high education, threatened abortion, chronic disease, and primipara appeared to use more Chinese herbal medicines than others in the sample. Postpartum women with high education, primipara, normal spontaneous delivery, and breastfeeding were found to use more Chinese herbal medicines; but women with pregnancy-related illness used less.

Conclusions: Chinese herbal medicines are frequently used by women during pregnancy and the postpartum period in Taiwan and those with high education and primipara used more such herbs. Due to limited safety information on these herbs, we would advise caution regarding their use either during pregnancy or postpartum breastfeeding period. Moreover, it is important for nurses/midwifes enquiring about such habits, and providing the adequate education to women during prenatal and postpartum care to prevent potential side effects.
\end{abstract}

(c) 2008 Elsevier Ltd. All rights reserved.

\footnotetext{
* Corresponding author. Tel.: +886 2 33668088; fax: +886 223582402.

E-mail address: pchen@ntu.edu.tw (P.-C. Chen).
}

\section{What is already known about the topic?}

- Pregnant women using traditional medicine or complementary and alternative medicine (TM/CAM) is gaining in popularity worldwide. 
- The prevalence rate of Chinese herbal medicines used by pregnant women was commonly in Taiwan and Chinese communities.

\section{What this paper adds}

- The current representative prevalence and risk factors of Chinese herbal medicine used by pregnant women in Taiwan.

- This research adds to measure the prevalence rate and risk factors of Chinese herbal medicines used by postpartum women.

\section{Introduction}

The use of herbal medicines during the perinatal period is becoming more common, especially in Chinese communities (Chen and Wang, 2000; Chuang et al., 2007; Forster et al., 2006; Gibson et al., 2001; Li, 2007; Nordeng and Havnen, 2004; Pinn and Pallett, 2002; Refuerzo et al., 2005; Tsui et al., 2001). Because use of all kinds of herbs during pregnancy or postpartum might produce potential adverse effects on mothers and fetuses, such practices raise concerns (Ernst, 2002; Tiran, 2003). Studies of these have shown inconclusive results depending on the different herbs used for different purposes in pregnancy (Chuang et al., 2006a,b; Holst et al., 2008; Vutyavanich et al., 2001). Furthermore, there is scarce evidence of developmental toxicity in animals (Chan et al., 2003; Jahnke et al., 2006; Tzeng et al., 1993). The adverse effect cannot be excluded, therefore, certain herbs are not recommended for pregnant women (Chan, 1993). The risk factors of herbal medicines used in pregnancy have been found to be diverse in different studies. Studies have shown that pregnant women with high maternal age (Holst et al., 2008; Nordeng and Havnen, 2005), high education (Gibson et al., 2001), who are white (Gibson et al., 2001), have a positive knowledge of and attitude toward herbs (Holst et al., 2008; Nordeng and Havnen, 2005), and have threatened abortion (Chuang et al., 2007) are more likely to use herbal medicines.

The Chinese postpartum custom of "doing the month" (Zuo yuezi), a month-long period of postpartum rest and recuperation, is common not only in Taiwan (Chen and Wang, 2000), but also in other places with Chinese communities (Cheng, 1997; Raven et al., 2007). The habit of using herbs by puerperal women is very common during this period (Chen and Wang, 2000; Hou et al., 2006; Hung, 2001). There is little evidence of herbs being used by women during the period after childbirth. One study showed that some Chinese medicine doctors had met women suffering adverse effects caused by the common herbs (Sheng-Hua-Tang) used for the postpartum recovery in Taiwan (Hou et al., 2006), and the another study showed that mothers taking another mixture of traditional Chinese herbs (Szu-Wu-Tang) during the postpartum period significantly increased the body burden of lead in their infants through breastfeeding (Chien et al., 2006). The mother's characteristics of being younger, having less education, and being primiparious (Kaewsarn et al., 2003) were factors related to traditional postpartum practices, and mothers and mothers-in-law were the most influential persons in recommending such behaviors (Chen and Wang, 2000; Kaewsarn et al., 2003).

There exists a general lack of research into the use of herbal medicines in pregnancy, let alone in the postpartum period. Hence, in the current study, the prevalence and risk factors of using Chinese herbal medicines during pregnancy and postpartum have been explored.

\section{Methods}

\subsection{Study population and sampling strategy}

The Taiwan Birth Cohort Study (TBCS), the first national birth cohort study in Taiwan, is a prospective longitudinal cohort study. In the current study, we used a multistage stratified systematic sampling design to obtain representative samples from the Taiwan national birth registration data in 2005. We ranked a total of 369 towns in Taiwan into 12 strata according to the administrative division (four strata) and the total fertility rate (three strata). Using the principle of proportion probability to size, we randomly sampled 90 towns out of 369 in Taiwan. All postpartum women and newborns, from these 90 towns were recruited, a total of 24,200 pairs.

\subsection{Data collection}

We conducted a home interview with the 24,200 postpartum women 6 months after their deliveries by using a structured questionnaire in the period from June 2005 to July 2006 . There were 2952 cases of loss to followup because of refusal to participate, moving home, incorrect addresses, infant deaths, and other miscellaneous reasons. A total of 21,248 postpartum women were interviewed, and the completed interview rate was thus $87.8 \%$.

\subsection{Variables}

Data were obtained from the interview questionnaire. The population was grouped by age: 19 and below; 20-34; and 35 or above. The educational levels were stratified into three groups: university and college or above; senior high school; and junior high school and below. The classification of occupation was summarized into two groups: occupation and no occupation. Family income per month was defined as the total parental income per month within four categories expressed in new Taiwan dollars (NT\$, new Taiwan dollars, 1 US\$ $\approx 30.5$ NT\$ in 2008): 30,000 or lower, $30,001-100,000,100,001-200,000$, and over 200,000.

Maternal chronic diseases, pregnancy-related illness, and types of delivery were obtained from the interview questionnaire. Maternal chronic diseases included hypertension, heart disease, diabetes mellitus, asthma, and allergy diseases. Gestational hypertension, diabetes, hyperemesis gravidarum, infection and fever during pregnancy, and eclampsia were counted as pregnancy-related illness. Types of delivery were normal spontaneous delivery or cesarean section. In addition, the questionnaires also asked whether 
Table 1

Prevalence of Chinese herbal medicines used during pregnancy and postpartum.

\begin{tabular}{|c|c|c|c|}
\hline Chinese herbal medicine & Type & Ingredients & $N(\%)$ \\
\hline Pregnancy & & & $21,248(100.0)$ \\
\hline At least one & - & - & $7,136(33.6)$ \\
\hline An-Tai-Yin & Formula & $\begin{array}{l}\text { Fritillariae Bulbus, Zingiber Rhizoma, Angelicae Radix, Glycyrrhizae Radix, } \\
\text { Ligustici Rhizoma, Paeoniae Lactiflorae Radix, Astragali Radix, Notopterygii Rhizoma, } \\
\text { Magnoliae Cortex, Schizonepetae Herba, Citri Immaturus Fructus, Artemisiae } \\
\text { Argyi Folium, and Cuscutae Semen }\end{array}$ & $2,871(13.5)$ \\
\hline Pearl powder & Single & Margarita & $2,529(11.9)$ \\
\hline Huanglian & Single & Coptidis Rhizoma & $2,261(10.6)$ \\
\hline Szu-Wu-Tang & Formula & Rehmanniae Radix, Paeoniae Radix, Angelicae Sinensis Radix, and Chuanxiong Rhizoma & $1,335(6.3)$ \\
\hline Ginseng & Single & Ginseng Radix & $948(4.5)$ \\
\hline \multicolumn{4}{|l|}{ Postpartum } \\
\hline Any one & - & - & $18,633(87.7)$ \\
\hline Sheng-Hua-Tang & Formula & $\begin{array}{l}\text { Angelicae Radix, Ligustici Rhizoma, Semen Persicae, Zingiberis Rhizoma, and } \\
\text { Glycyrrhizae Radix }\end{array}$ & $17,543(82.6)$ \\
\hline Szu-Wu-Tang & Formula & Rehmanniae Radix, Paeoniae Radix, Angelicae Sinensis Radix, and Chuanxiong Rhizoma & $9,522(44.8)$ \\
\hline
\end{tabular}

Values in parentheses are percentages.

there was a threatened abortion during pregnancy, and if the mother's breastfed or went to a postpartum recovery center (Zuo-yuezi center).

Information related to infants such as gender, birth weight, and gestational weeks was obtained from the national Taiwan birth register. Low birth weight refers to babies with birthweight below $2500 \mathrm{~g}$ and preterm delivery to babies born before 37 completed weeks (259 days) of gestation.

Chinese herbal medicine was defined as any botanical material or preparation with therapeutic or other human health benefits, which contains either raw or processed ingredients from one or more plants. Materials of inorganic or animal origin may also be present (WHO, 2000). The content of Chinese herbal medicines used during pregnancy and postpartum period were classified according to a previous study in Taiwan (Chuang et al., 2005). They were grouped as An-Tai-Yin, Pearl powder, Huanglian, Szu-WuTang, Ginseng, and others during pregnancy; Sheng-HuaTang and Szu-Wu-Tang during the postpartum period, as shown in Table 1. In brief, data were gathered on common Chinese herbal medicines according to four different time periods: the first 3 months, after 3 months, within 1 month after childbirth (Zuo yuezi period), and beyond 1 month after childbirth.

\subsection{Statistical analysis}

Multiple logistic regression was performed to estimate odds ratios (ORs) and the $95 \%$ confidence intervals (CIs) of the dichotomous outcomes after adjusting for the potential confounding variables of maternal age, education, occupation, family income per month (NT\$), threatened abortion, pregnancy-related illness, chronic diseases, and parity. In addition to these potential confounders, type of delivery, breastfeeding, and postpartum recovery centers were added to estimate the odds ratios of women using herbal medicines after childbirth. This statistical analysis was performed using SPSS for Windows, Release 11.0.

\section{Results}

\subsection{Prevalence of Chinese herbal medicines used during pregnancy and postpartum}

The prevalence of Chinese herbal medicines use during pregnancy and the postpartum period was shown in Table 1. Among 21,248 women in the current study, 7136 (33.6\%) and 18,633 (87.7\%) reported using Chinese herbal medicines during pregnancy and the postpartum period, respectively. The five most commonly used Chinese herbal medicines used during pregnancy were An-Tai-Yin (13.5\%), Pear powder (11.9\%), Huanglian (10.6\%), Szu-Wu-Tang (6.3\%), and Ginseng (4.5\%). Sheng-Hua-Tang (82.6\%) and Szu-Wu-Tang (44.8\%) were the most commonly used during the postpartum period.

\subsection{Characteristics of the study subjects}

The characteristics of the subjects we interviewed were summarized in Tables 2 and 3, which also showed the results of multiple logistic regressions for various risk factors of using Chinese herbal medicines during pregnancy and the postpartum period.

Most of the subjects were 20-34 years old (85.3\%). There was a predominance of university or graduate education (45.1\%), and most of subjects had a job (61.0\%); $77.1 \%$ of the families had a month income of $30,000-$ 100,000 NT\$. The prevalence of threatened abortion, pregnancy-related illness and maternal chronic diseases during the current pregnancy was $24.9 \%, 31.5 \%$, and $19.4 \%$, respectively. About half of the mothers were primipara (50.4\%). 66.6\% women had normal spontaneous delivery (NSD), and $82.1 \%$ breastfed their child.

\subsection{Risk factors of using Chinese herbal medicines}

The risk factors of pregnancy-related use of Chinese herbal medicines were shown in Table 2. After adjustment for all other variables, pregnant women aged 20-34 (OR 
Table 2

Risk factors of pregnancy-related Chinese herbal medicine use among pregnant women in Taiwan.

\begin{tabular}{|c|c|c|c|c|}
\hline \multirow[t]{3}{*}{ Risk factors } & \multicolumn{4}{|c|}{ Chinese Herbal Medicines Use } \\
\hline & Yes $(N=7136)$ & No $(N=14,112)$ & cOR $(95 \% \mathrm{CI})$ & aOR $(95 \% \mathrm{CI})$ \\
\hline & No. (\%) & No. (\%) & & \\
\hline Maternal age (years) & & & $P<0.001$ & $P=0.010$ \\
\hline$\leq 19$ & $133(1.9)$ & $304(2.2)$ & $1.00(0.80,1.25)$ & $1.08(0.85,1.35)$ \\
\hline $20-34$ & $6,186(86.7)$ & $11,942(84.6)$ & $1.18(1.08,1.29)^{\#}$ & $1.15(1.05,1.26)^{* *}$ \\
\hline$\geq 35^{a}$ & $817(11.4)$ & $1,866(13.2)$ & 1.00 & 1.00 \\
\hline Maternal education & & & $P<0.001$ & $P<0.001$ \\
\hline Junior high school- ${ }^{a}$ & 776 (10.9) & $2,372(16.8)$ & 1.00 & 1.00 \\
\hline Senior high school & $3,000(42.0)$ & $5,519(39.1)$ & $1.66(1.52,1.82)^{\#}$ & $1.58(1.44,1.74)^{\#}$ \\
\hline University + & $3,360(47.1)$ & $6,221(44.1)$ & $1.65(1.51,1.81)^{\#}$ & $1.52(1.34,1.68)^{\#}$ \\
\hline Maternal occupation & & & $P<0.001$ & $P=0.215$ \\
\hline $\mathrm{No}^{\mathrm{a}}$ & $2,639(37.0)$ & $5,654(40.1)$ & 1.00 & 1.00 \\
\hline Yes & $4,497(63.0)$ & $8,458(59.9)$ & $1.14(1.07,1.21)^{\#}$ & $1.04(0.98,1.11)$ \\
\hline Family income per month (NT\$) & & & $P=0.002$ & \\
\hline$\leq 30,000^{\mathrm{a}}$ & $752(10.5)$ & $1,732(12.3)$ & 1.00 & - \\
\hline $30,001-100,000$ & $5,600(78.5)$ & $10,781(76.4)$ & $1.20(1.09,1.31)^{\#}$ & \\
\hline $100,001-200,000$ & $689(9.7)$ & $1,403(9.9)$ & $1.13(0.99,1.28)$ & \\
\hline$>200,000$ & $96(1.3)$ & $196(1.4)$ & $1.12(0.86,1.45)$ & \\
\hline Threatened abortion & & & $P<0.001$ & $P<0.001$ \\
\hline $\mathrm{No}^{\mathrm{a}}$ & $4,901(68.7)$ & $11,063(78.4)$ & 1.00 & 1.00 \\
\hline Yes & $2,235(31.3)$ & $3,049(21.6)$ & $1.66(1.55,1.76)^{\#}$ & $1.61(1.51,1.72)^{\#}$ \\
\hline Pregnancy-related illness & & & $P=0.027$ & $P=0.309$ \\
\hline $\mathrm{No}^{\mathrm{a}}$ & $4,814(67.5)$ & $9,731(69.0)$ & 1.00 & 1.00 \\
\hline Yes & $2,322(32.5)$ & $4,381(31.0)$ & $1.07(1.01,1.14)^{*}$ & $1.03(0.97,1.10)$ \\
\hline Maternal chronic diseases & & & $P<0.001$ & $P=0.002$ \\
\hline $\mathrm{No}^{\mathrm{a}}$ & $5,628(78.9)$ & $11,492(81.4)$ & 1.00 & 1.00 \\
\hline Yes & $1,508(21.1)$ & $2,620(18.6)$ & $1.18(1.10,1.26)^{\#}$ & $1.12(1.04,1.20)^{* *}$ \\
\hline Parity & & & $P<0.001$ & $P<0.001$ \\
\hline 0 & $3,877(54.3)$ & $6,836(48.4)$ & $1.33(1.20,1.46)^{\#}$ & $1.25(1.13,1.38)^{\#}$ \\
\hline 1 & $2,549(35.7)$ & $5,617(39.8)$ & $1.06(0.96,1.17)$ & $1.01(0.91,1.12)$ \\
\hline$\geq 2^{\mathrm{a}}$ & $710(9.9)$ & $1,659(11.8)$ & 1.00 & 1.00 \\
\hline
\end{tabular}

Values in parentheses are percentages. Considering the high correlation between maternal education and family income per month (NT\$) (data not shown), we excluded the factor of family income per month when we estimated the adjusted odds ratio.

Abbreviations: cOR, crude odd ratio; aOR, adjusted odd ratio; $\mathrm{CI}$, confidence interval; NT\$, new Taiwan dollars.

a Reference category.

${ }^{*} P<0.05$.

** $P<0.01$.

\# $P<0.001$.

1.15; 95\% CI 1.05, 1.26), with high education (senior high school: OR 1.58; 95\% CI 1.44, 1.74; university or over: OR $1.52 ; 95 \%$ CI $1.34,1.68$ ), threatened abortion (OR 1.61; 95\% CI 1.51, 1.72), chronic disease (OR 1.12; 95\% CI 1.04, 1.20), and primipara (OR $1.25 ; 95 \% \mathrm{CI} 1.13,1.38$ ) used more Chinese herbal medicines.

Table 3 showed the risk factors of using Chinese herbal medicines during postpartum. After adjustment for all other variables, postpartum women with high education (senior high school: OR 1.86; 95\% CI 1.67, 2.08; university or over: OR 2.23 ; 95\% CI 1.97, 2.53), primipara (OR 1.15; 95\% CI 1.00, 1.31 ), normal spontaneous delivery (OR 1.58 ; $95 \%$ CI 1.45 , 1.73), and breastfeeding (OR 1.16; 95\% CI 1.04, 1.28) used more Chinese herbal medicines, but those with pregnancyrelated illness (OR 0.88; 95\% CI 0.81, 0.96) used less.

\section{Discussion}

We found high overall use of Chinese herbal medicines among Taiwanese women during pregnancy
(33.6\%), and postpartum (87.7\%). Before reaching any further conclusions, the meaning of this study for a nursing/midwifery audience, and the representativeness of the sampling and validity of measurements need to be discussed.

Nurses can play an important role in the risk reduction of drug safety. In October 2004, WHO launched the World Alliance for Patient Safety in response to a World Health Assembly Resolution (2002) urging WHO and Member States to pay the closest possible attention to the problem of patient safety (WHO, 2004). Drug safety is an important part of patient safety, thus the important role of nursing in pharmacovigilance are followed with interests (Lata et al., 2004; Morrison-Griffiths et al., 2003; Ulfvarson et al., 2007). For pregnant or postpartum women, nurses or midwives are in a key position to evaluate adverse drug events or educate patients the correct medical information because of their direct patient care activities during prenatal or postpartum periods. Lacking the current representative data, the results of our study could provide 
Table 3

Risk factors of Chinese herbal medicine use during postpartum in Taiwan.

\begin{tabular}{|c|c|c|c|c|}
\hline \multirow[t]{3}{*}{ Risk factors } & \multicolumn{4}{|c|}{ Chinese herbal medicines use } \\
\hline & Yes $(N=18,633)$ & No $(N=2,615)$ & $\operatorname{cOR}(95 \% \mathrm{CI})$ & aOR $(95 \% \mathrm{CI})$ \\
\hline & No. $(\%)$ & No. $(\%)$ & & \\
\hline $\begin{array}{l}\text { Maternal age (years) } \\
\leq 19 \\
20-34 \\
\geq 35^{\mathrm{a}}\end{array}$ & $\begin{array}{r}355(1.9) \\
15,940(85.5) \\
2,338(12.5)\end{array}$ & $\begin{aligned} & 82(3.1) \\
& 2,188(83.7) \\
& 345(13.2)\end{aligned}$ & $\begin{array}{l}P<0.001 \\
0.64(0.49,0.83)^{\#} \\
1.08(0.95,1.21) \\
1.00\end{array}$ & $\begin{array}{l}P=0.330 \\
0.87(0.65,1.15) \\
1.04(0.91,1.18) \\
1.00\end{array}$ \\
\hline $\begin{array}{l}\text { Maternal education } \\
\text { Junior high school-a } \\
\text { Senior high school } \\
\text { University }+\end{array}$ & $\begin{array}{l}2,469(13.3) \\
7,472(40.1) \\
8,692(46.6)\end{array}$ & $\begin{array}{r}679(26.0) \\
1,047(40.0) \\
889(34.0)\end{array}$ & $\begin{array}{l}P<0.001 \\
1.00 \\
1.96(1.76,2.18)^{\#} \\
2.68(2.41,3.00)^{\#}\end{array}$ & $\begin{array}{l}P<0.001 \\
1.00 \\
1.86(1.67,2.08)^{\#} \\
2.23(1.97,2.53)^{\#}\end{array}$ \\
\hline $\begin{array}{l}\text { Maternal occupation } \\
\mathrm{No}^{\mathrm{a}} \\
\text { Yes }\end{array}$ & $\begin{array}{r}6,993(37.5) \\
11,640(62.5)\end{array}$ & $\begin{array}{l}1,300(49.7) \\
1,315(50.3)\end{array}$ & $\begin{array}{l}P<0.001 \\
1.00 \\
1.65(1.52,1.79)^{\#}\end{array}$ & $\begin{array}{l}P<0.001 \\
1.00 \\
1.32(1.21,1.44)^{* *}\end{array}$ \\
\hline $\begin{array}{l}\text { Family income per month (NT\$) } \\
\leq 30,000^{\mathrm{a}} \\
30,001-100,000 \\
100,001-200,000 \\
>200,000\end{array}$ & $\begin{array}{r}2,005(10.8) \\
14,474(77.7) \\
1,901(10.2) \\
253(1.4)\end{array}$ & $\begin{array}{r}479(18.3) \\
1,907(72.9) \\
191(7.3) \\
38(1.5)\end{array}$ & $\begin{array}{l}P<0.001 \\
1.00 \\
1.81(1.62,2.03)^{\#} \\
2.38(1.99,2.84)^{\#} \\
1.59(1,12,2,27)^{*}\end{array}$ & - \\
\hline $\begin{array}{l}\text { Pregnancy-related illness } \\
\mathrm{No}^{\mathrm{a}} \\
\text { Yes }\end{array}$ & $\begin{array}{r}12,852(69.0) \\
5,781(31.0)\end{array}$ & $\begin{array}{r}1,693(64.7) \\
922(35.3)\end{array}$ & $\begin{array}{l}P<0.001 \\
1.00 \\
0.83(0.76,0.90)^{\#}\end{array}$ & $\begin{array}{l}P=0.004 \\
1.00 \\
0.88(0.81,0.96)^{* *}\end{array}$ \\
\hline $\begin{array}{l}\text { Maternal chronic diseases } \\
\mathrm{No}^{\mathrm{a}} \\
\text { Yes }\end{array}$ & $\begin{array}{r}14,955(80.3) \\
3,678(19.7)\end{array}$ & $\begin{array}{r}2,165(82.8) \\
450(17.2)\end{array}$ & $\begin{array}{l}P=0.002 \\
1.00 \\
1.18(1.06,1.32)^{* *}\end{array}$ & $\begin{array}{l}P=0.068 \\
1.00 \\
1.11(0.99,1.24)\end{array}$ \\
\hline $\begin{array}{l}\text { Parity } \\
0 \\
1 \\
\geq 2^{\mathrm{a}}\end{array}$ & $\begin{array}{l}9,469(50.8) \\
7,157(38.4) \\
2,007(10.8)\end{array}$ & $\begin{array}{r}1,244(47.6) \\
1,009(38.6) \\
362(13.8)\end{array}$ & $\begin{array}{l}P<0.001 \\
1.37(1.21,1.56)^{\#} \\
1.28(1.12,1.46)^{\#} \\
1.00\end{array}$ & $\begin{array}{l}P=0.134 \\
1.15(1.00,1.31)^{*} \\
1.11(0.97,1.26) \\
1.00\end{array}$ \\
\hline $\begin{array}{l}\text { Type of delivery } \\
\text { NSD } \\
\text { C/S }\end{array}$ & $\begin{array}{r}12,629(67.8) \\
6,004(32.2)\end{array}$ & $\begin{array}{l}1,513(57.9) \\
1,102(42.1)\end{array}$ & $\begin{array}{l}P<0.001 \\
1.53(1.41,1.67)^{\#} \\
1.00\end{array}$ & $\begin{array}{l}P<0.001 \\
1.58(1.45,1.73)^{\#} \\
1.00\end{array}$ \\
\hline $\begin{array}{l}\text { Breastfeeding } \\
\text { Ever } \\
\text { Never }^{\mathrm{a}}\end{array}$ & $\begin{array}{r}15,424(82.8) \\
3,209(17.2)\end{array}$ & $\begin{array}{r}2,027(77.5) \\
588(22.5)\end{array}$ & $\begin{array}{l}P<0.001 \\
1.39(1.26,1.54)^{\#} \\
1.00\end{array}$ & $\begin{array}{l}P=0.006 \\
1.16(1.04,1.28)^{* *} \\
1.00\end{array}$ \\
\hline $\begin{array}{l}\text { Postpartum recovery centers } \\
\text { (zuo-yuezi center) }\end{array}$ & & & $P<0.001$ & $P=0.059$ \\
\hline Yes & $1,428(7.7)$ & $137(5.2)$ & $1.50(1.25,1.80)^{\#}$ & $1.20(0.99,1.44)$ \\
\hline $\mathrm{No}^{\mathrm{a}}$ & $17,205(92.3)$ & $2,478(94.8)$ & 1.00 & 1.00 \\
\hline
\end{tabular}

Values in parentheses are percentages. Considering the high correlation between maternal education and family income per month (NT\$) (data not shown), we excluded the factor of family income per month when we estimated the adjusted odds ratio.

Abbreviations: cOR, crude odd ratio; aOR, adjusted odd ratio; CI, confidence interval; NT\$, new Taiwan dollars; NSD, normal spontaneous delivery; C/S, cesarean section.

${ }^{\text {a }}$ Reference category.

${ }^{*} P<0.05$.

${ }^{* *} P<0.01$

\# $P<0.001$.

the information related the women using Chinese herbal medicines during prenatal or postpartum periods.

The characteristics of non-responders need to be considered. There were no significant differences with the characteristics of parents' age, type of delivery, and gender of newborn between the responders and nonresponders. However, the rate of low birth weight (9.6\%) and preterm delivery births (11.0\%) in the non-responders was significantly higher than that in the responders $(6.9 \%$ and $8.4 \%$, respectively). Consequently, a potential selection bias might exist because some low birth weight or preterm babies were not recruited to respond. It might, therefore, have led us to underestimate the prevalence of using Chinese herbal medicines if low birth weight or preterm babies were related to the use of such medicines during pregnancy.

The prevalence of herbs used during pregnancy (33.6\%) in this current study was similar to recent studies in Norway (36\%) (Nordeng and Havnen, 2004) and Taiwan (24.1\%) (Chuang et al., 2007), but lower than a mid-1980s Taiwan survey (42.3\%) (Chuang et al., 2005). This shows that pregnant women in Taiwan about using traditional Chinese herbal medicines seem to have become more cautious. 
The most common herb used during pregnancy was An-Tai-Yin (13.5\%), and then about $10 \%$ of pregnant women used Pearl powder or Huanglian. In addition, 1596 (7.5\%) of pregnant women used An-Tai-Yin in the first trimester, and 878 of them reported no symptom of threatened abortion (data not shown). Traditional wisdom in Taiwan is that An-Tai-Yin prevents spontaneous abortion, and indeed the name implies this in Chinese. However, according to an ancient Chinese book (Fu et al., 1999), the main effect of An-Tai-Yin is to help pregnant women deliver smoothly. Hence, the most appropriate time to use An-Tai-Yin is in the third trimester. In addition, around $2 \%$ of women without any indication used other herbs during the first trimester. Our previous study showed that herbs used during the first trimester caused a high risk of congenital malformation (Chuang et al., 2006a). Furthermore, little information is available on the consumption and safety of Pearl powder and Huanglian. Hence, such habits during prenatal care must be enquired and this requires further research.

The very widespread use of herbs during postpartum (87.7\%) found in our study is consistent with another study in Taiwan which reported $94.4 \%$ postpartum women using Sheng-Hua-Tang (Chen and Wang, 2000). Sheng-Hua-Tang (82.6\%) and Szu-Wu-Tang (44.8\%) were the most commonly used herbs in the postpartum period. According to the traditional Taiwanese postpartum custom of "doing the month" (Zuo yuezi), women are taught by their mothers or mother-in-laws to take Chinese herbal medicines such as Sheng-Hua-Tang (Chen and Wang, 2000) and Szu-Wu-Tang to recuperate from the delivery. Such a practice usually lasts for 1 month from the date of childbirth. In our study about $2 \%$ of women used Sheng-Hua-Tang for more than 1 month after childbirth, longer than the recommendation of the Chinese medicine doctors, and this might cause vaginal hemorrhage (Hou et al., 2006). Furthermore, infants breastfed by postpartum women using Szu-Wu-Tang had the risk of elevated lead, because of the herbs contaminated by lead (Chien et al., 2006). Hence, this practice should be prescribed by Chinese medicine doctors for postpartum recovery.

Similar to previous studies in Taiwan (Chuang et al., 2005, 2007), pregnant women in our survey with a threatened abortion and primipara used more Chinese herbal medicines. Other factors related to using more herbs during pregnancy were women aged 20-34, chronic diseases, and high education. The finding that women with high education used more herbs was opposite to that in the previous 1980s study, which showed that low socio-economic women used more herbs (Chuang et al., 2005). One of the most probable reasons for such difference is that the kinds of herbs used during pregnancy have been modified in the current era, especially Pearl powder. While the most common used herbs during pregnancy (An-Tai-Yin, Huanglian, Szu-WuTang, and Ginseng) were similar in the mid-1980s survey (Chuang et al., 2005) and the current study, Pearl powder was rarely used several decades ago. In Taiwanese popular understanding, the function of Pearl powder is similar to that of Huanglian, to make the fetal skin beautiful. Our study showed the prevalence rates of Pearl powder use by pregnant women with education levels of university or over, senior high school, and junior high school or lower were $15.2 \%, 11.0 \%$, and $4.5 \%$, respectively (data not shown). Women with higher education thus used significantly more Pearl powder. This is perhaps because the price of Pearl powder is higher than Huanglian, hence, women in the high socio-economic level could better afford it.

In the current study, during postpartum, women with high education, primipara, normal spontaneous delivery, and breastfeeding used more herbs, but those with pregnancy-related illness diseases used less. This is consistent with the belief of gynecologists that ShengHua-Tang can help lochia discharge, and thus women with normal spontaneous delivery need to use more than women who had a cesarean section, during which the doctor had cleaned the lochia (Hou et al., 2006).

Ethical issues in the longitudinal follow up study are concerned. Firstly, the current study was approved by the Ethics Review Board of the National Taiwan University College of Public Health. Postcards were delivered to subjects, thus they can consider participating or dropping out, and participants are allowed to refuse any specific aspect of the study. Then, consents were obtained at enrollment and at each home-interview, and some variables must ethically be fed back to the participants. Specific situations such as people whose babies had died, had been adapted or had potential developmental problems, we provided the information of counseling or referrals.

\section{Conclusions}

Chinese herbal medicines are frequently used by women during pregnancy and the postpartum period in Taiwan and those with high education and primipara used more such herbs. Due to limited safety information on these herbs, we would advise caution regarding their use either during pregnancy or postpartum breastfeeding period. Moreover, it is important for nurses/midwifes enquiring about such habits, and providing the adequate education to women during prenatal and postpartum care to prevent potential side effects.

\section{Acknowledgements}

We thank the enduring support and assistance from Professor Tung-Liang Chiang, Institute of Health Policy and Management, National Taiwan University College of Public Health, Taipei, Taiwan; Professor Yueliang Leon Guo, Department of Environmental and Occupational Medicine, National Taiwan University Hospital and National Taiwan University College of Medicine, Taipei, Taiwan; Professor Meng-Chin Lee, Institute of Medicine, Professor Hui-Sheng Lin, School of Public Health, Chung Shan Medical University. Taichung, Taiwan; and Professor Bih-Ching Shu, Institutes of Allied Health Sciences, College of Medicine, National Cheng Kung University, Tainan, Taiwan. 


\section{Appendix A}

Questions for collecting the information of women used Chinese herbal medicines during pregnancy and postpartum periods.

Part 1: Questions of Chinese herbal medicines used during the pregnancy

1. Did you use An-Tai-Yin during the pregnancy?

\section{No (Skip to the next question.)}

Yes

a. When did you use An-Tai-Yin during the pregnancy?

$\square$ during the first trimester

$\square$ during the second and third trimesters

b. How many times did you use An-Tai-Yin during the pregnancy?

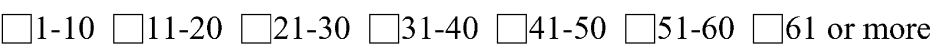

2. Did you use Pearl powder during the pregnancy?

\section{No (Skip to the next question.)}

Yes

a. When did you use Pearl powder during the pregnancy?

during the first trimester

during the second and third trimesters

b. How many times did you use Pearl powder during the pregnancy?

$\square$ 1-10 $\square$ 11-20 $\square$ 21-30 $\square$ 31-40 $\square$ 41-50 $\square$ 51-60 $\square 61$ or more

3. Did you use Huanglian during the pregnancy?

\section{No (Skip to the next question.)}

Yes

a. When did you use Huanglian during the pregnancy?

$\square$ during the first trimester

during the second and third trimesters

b. How many times did you use Huanglian during the pregnancy?

$\square$ 1-10 $\square$ 11-20 $\square$ 21-30 $\square$ 31-40 $\square$ 41-50 $\square$ 51-60 $\square 61$ or more

4. Did you use Szu-Wu-Tang during the pregnancy?

\section{No (Skip to the next question.)}

Yes

a. When did you use Szu-Wu-Tang during the pregnancy?

during the first trimester

during the second and third trimesters

b. How many times did you use Szu-Wu-Tang during the pregnancy?

$\square$ 1-10 $\square$ 11-20 $\square$ 21-30 $\square$ 31-40 $\square$ 41-50 $\square$ 51-60 $\square 61$ or more

5. Did you use Ginseng during the pregnancy?

\section{No (Skip to the next question.)}

Yes 
a. When did you use Ginseng during the pregnancy?

$\square$ during the first trimester

$\square$ during the second and third trimesters

b. How many times did you use Ginseng during the pregnancy?

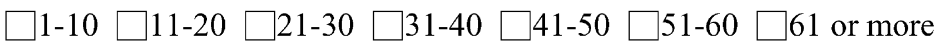

6. Did you use other Chinese herbal medicines during the pregnancy?

\section{No (Skip to the next question.)}

Yes

a. When did you use other Chinese herbal medicines during the pregnancy?

$\square$ during the first trimester

$\square$ during the second and third trimesters

b. How many times did you use the Chinese herbal medicine during the pregnancy?

$\square 1-10 \square 11-20 \square 21-30 \square$ 31-40 $\square 41-50 \square$ 51-60 $\square 61$ or more

\section{Part 2: Questions of Chinese herbal medicines used during the postpartum period}

1. Did you use Sheng-Hua-Tang during the postpartum period?

$\square$ No (Skip to the next question.)

Yes

a. When did you use Sheng-Hua-Tang during the postpartum period?

$\square$ during Zuo yuezi period

$\square$ after Zuo yuezi period

b. How many times did you use Sheng-Hua-Tang during the postpartum period?

$\square$ 1-10 $\square$ 11-20 $\square 21-30 \square$ 31-40 $\square$ 41-50 $\square$ 51-60 $\square 61$ or more

2. Did you use Szu-Wu-Tang during the postpartum period in the current pregnancy?

\section{No (Skip to the next question.)}

Yes

a. When did you use Szu-Wu-Tang during the postpartum period?

$\square$ during Zuo yuezi period

$\square$ after Zuo yuezi period

b. How many times did you use Szu-Wu-Tang during the postpartum period?

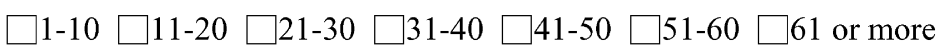

3. Did you use other Chinese herbal medicines during the postpartum period?

No (Skip to the next question.)

Yes

a. When did you use other Chinese herbal medicine during the postpartum period?

$\square$ during Zuo yuezi period

$\square$ after Zuo yuezi period

b. How many times did you use other Chinese herbal medicine during the postpartum period?
$\square 1-10$
$11-20$
$\square 21-30$
$\square 31-40$ $\square 41-50$ $\square 51-60$ 61 or more 
Conflict of interest: The authors declare that they have no competing interests that might affect this report.

Ethical approval: This study was approved by the Ethics Review Board of the National Taiwan University College of Public Health.

Funding: This study was based on the data from the Taiwan Birth Cohort Study Database and supported by the grants (BHP-PHRC-92-4, DOH93-HP-1702 and DOH94-HP1702) from the Bureau of Health Promotion, Department of Health, Taiwan.

\section{References}

Chan, E., 1993. Displacement of bilirubin from albumin by berberine. Biology of the Neonate 63 (4), 201-208.

Chan, L.Y., Chiu, P.Y., Lau, T.K., 2003. An in-vitro study of ginsenoside Rb1induced teratogenicity using a whole rat embryo culture model. Human Reproduction 18 (10), 2166-2168.

Chen, L.L., Wang, C.C., 2000. Attitude and behavior towards postpartum recuperation in traditional Chinese medicines. Journal of Nursing Research (ROC) 8 (1), 49-58.

Cheng, N.F., 1997. Chinese zuo yuezi (sitting in for the first month of the postnatal period) in Scotland. Midwifery 13 (2), 55-65.

Chien, L.C., Yeh, C.Y., Lee, H.C., Chao, H.J., Shieh, M.J., Han, B.C., 2006. Effect of the mother's consumption of traditional Chinese herbs on estimated infant daily intake of lead from breast milk. Science of the Total Environment 354 (2-3), 120-126.

Chuang, C.H., Lai, J.N., Wang, J.D., Chang, P.J., Chen, P.C., 2005. Prevalence and Related Factors of Chinese Herbal Medicine Use in Pregnant Women of Taipei 1985-1987. Taiwan Journal of Public Health 24 (4), 335-347.

Chuang, C.H., Doyle, P., Wang, J.D., Chang, P.J., Lai, J.N., Chen, P.C., $2006 a$. Herbal medicines used during the first trimester and major congenital malformations-an analysis of data from a pregnancy cohort study. Drug Safety 29 (6), 537-548.

Chuang, C.H., Lai, J.N., Wang, J.D., Chang, P.J., Chen, P.C., 2006b. Use of Coptidis Rhizoma and foetal growth-a follow-up study of 9895 pregnancies. Pharmacoepidemiology and Drug Safety 15 (3), 185-192.

Chuang, C.H., Hsieh, W.S., Guo, Y.L., Lin, S.H., Lin, S.J., Chen, P.C., 2007. Chinese herbal medicines used in pregnancy: a population-based survey in Taiwan. Pharmacoepidemiology and Drug Safety 16 (4), 464-468.

Ernst, E., 2002. Herbal medicinal products during pregnancy: are they safe? British Journal of Obstetrics and Gynaecology 109 (3), 227-235.

Forster, D.A., Denning, A., Wills, G., Bolger, M., McCarthy, E., 2006. Herbal medicine use during pregnancy in a group of Australian women. BMC Pregnancy Childbirth 6, 21, doi:10.1186/1471-2393-6-21.

Fu, Q.Z., Yang, S.Z., Liu, D.W., 1999. Fu Qing-zhus Gynecology. Blue Poppy Press, Boulder, CO.

Gibson, P.S., Powrie, R., Star, J., 2001. Herbal and alternative medicine use during pregnancy: a cross-sectional survey. Obstetrics and Gynecology 97 (4 Suppl. 1), S44-S45.

Holst, L., Nordeng, H., Haavik, S., 2008. Use of herbal drugs during early pregnancy in relation to maternal characteristics and pregnancy outcome. Pharmacoepidemiology and Drug Safety 17 (2), 151-159.
Hou, Y.C., You, J.S., Chang, H.H., 2006. The differences of sheng-hua-tang on the postpartum recovery among normal spontaneous delivery, cesarean section and artifical abortion-the analytic studies of 34 gynecological experts' experiences in traditional Chinese medicine. Journal of Chinese Medicine (ROC) 17 (4), 127-134.

Hung, P., 2001. Traditional Chinese customs and practices for the postnatal care of Chinese mothers. Complementary Therapies in Nursing and Midwifery 7 (4), 202-206.

Jahnke, G.D., Price, C.J., Marr, M.C., Myers, C.B., George, J.D., 2006. Developmental toxicity evaluation of berberine in rats and mice. Birth Defects Research (Part B) 77 (3), 195-206.

Kaewsarn, P., Moyle, W., Creedy, D., 2003. Traditional postpartum practices among Thai women. Journal of Advanced Nursing 41 (4), 319-418.

Lata, P.F., Mainhard, M., Johnson, C.A., 2004. Impact of nurse case manager-pharmacist collaboration on adverse-drug-event reporting. American Journal of Health-System Pharmacy 61 (5), 483-487.

Li, D.Z., 2007. Use of traditional Chinese herbal medicines during early pregnancy in mainland China. Pharmacoepidemiology and Drug Safety 16 (8), 942-945.

Morrison-Griffiths, S., Walley, T.J., Park, B.K., Breckenridge, A.M., Pirmohamed, M., 2003. Reporting of adverse drug reactions by nurses. Lancet 361 (9366), 1347-1348.

Nordeng, H., Havnen, G.C., 2004. Use of herbal drugs in pregnancy: a survey among 400 Norwegian women. Pharmacoepidemiology and Drug Safety 13 (6), 371-380.

Nordeng, H., Havnen, G.C., 2005. Impact of socio-demographic factors, knowledge and attitude on the use of herbal drugs in pregnancy. Acta Obstetricia et Gynecologica Scandinavica 84 (1), 26-33.

Pinn, G., Pallett, L., 2002. Herbal medicine in pregnancy. Complementary Therapies in Nursing and Midwifery 8 (2), 77-80.

Raven, J.H., Chen, Q., Tolhurst, R.J., Garner, P., 2007. Traditional beliefs and practices in the postpartum period in Fujian Province, China: a qualitative study. BMC Pregnancy Childbirth 7, 8, doi:10.1186/ 1471-2393-7-8.

Refuerzo, J.S., Blackwell, S.C., Sokol, R.J., et al., 2005. Use of over-thecounter medications and herbal remedies in pregnancy. American Journal of Perinatology 22 (6), 321-324.

Tiran, D., 2003. The use of herbs by pregnant and childbearing women: a risk-benefit assessment. Complementary Therapies in Nursing and Midwifery 9 (4), 176-181.

Tsui, B., Dennehy, C.E., Tsourounis, C., 2001. A survey of dietary supplement use during pregnancy at an academic medical center. American Journal of Obstetrics \& Gynecology 185 (2), 433-437.

Tzeng, C.R., Yang, L.L., Chien, L.W., Chang, S.R., Chen, A.C., 1993. Effect of traditional Chinese medicine on mouse embryo development. Journal of Taipei Medical College 22 (1), 43-46.

Ulfvarson, J., Mejyr, S., Bergman, U., 2007. Nurses are increasingly involved in pharmacovigilance in Sweden. Pharmacoepidemiology and Drug Safety 16 (5), 532-537.

Vutyavanich, T., Kraisarin, T., Ruangsri, R.A., 2001. Ginger for nausea and vomiting in pregnancy: a randomised, double-masked, placebo-controlled trial. Obstetrics and Gynecology 97 (4), 577-582.

World Health Organization, 2000. General guidelines for methodologies on research and evaluation of Traditional Medicines. World Health Organization, Geneva, p. 27 (document reference WHO/EDM/TRM/ 2000.1).

World Health Organization, 2004. World Alliance for Patient Safety. Available from http://www.who.int/patientsafety/en/ (accessed on 10.10.08). 